\title{
Efecto de los errores en la inseminación con semen congelado sobre la morfofisiología espermática bovina
}

\author{
The effect of errors regarding frozen semen insemination on \\ bovine sperm morphophysiology
}

\author{
Efeito dos erros na inseminação com sêmen congelado na \\ morfofisiologia esperma bovino
}

\author{
Norberto Villa-Duque ${ }^{1}$; Julián A. Valencia-Giraldo ${ }^{2 *}$; Germán Gómez-Londoño ${ }^{3 *}$; \\ Francisco J. Henao-Uribe ${ }^{3 *}$
}

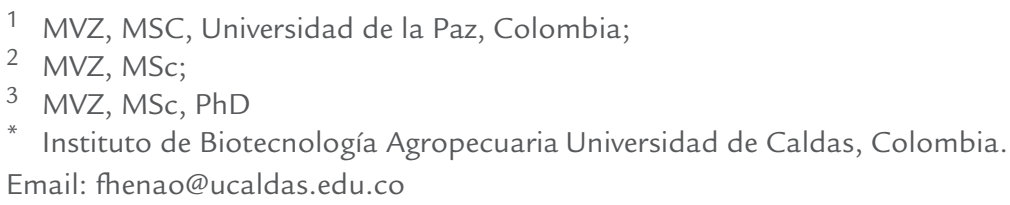

Recibido: febrero 2 de 2015

Aceptado: mayo 25 de 2016

\begin{abstract}
Resumen
En el presente trabajo se evaluó la criopreservación a $-196^{\circ} \mathrm{C}$, la descongelación y la aplicación del semen, en 32 ganaderías bovinas del centro de Colombia, y se estudió, in vitro, el efecto de los errores encontrados sobre la integridad de las membranas y la morfología espermáticas. La recolección de información en fincas se realizó mediante formulario específico. El estudio in vitro se ejecutó en el Instituto de Biotecnología Agropecuaria de la Universidad de Caldas (Manizales, Colombia), y consistió en someter pajillas comerciales de $0.5 \mathrm{ml}\left(30 \times 10^{6}\right.$ epzs), de toros Holstein, Jersey y Pardo Suizo, a la técnica instrumental convencional y a cinco modificaciones de la misma (errores más frecuentes), mediante un arreglo factorial 6x3 en diseño completamente al azar. En ninguna de las fincas evaluadas se aplicó correctamente la práctica de la inseminación artificial (IA). Los errores más frecuentes fueron: deterioro de la estructura externa y condiciones inadecuadas de almacenamiento de los tanques, almacenamiento de pajillas en la canastilla, y manipulación de pajillas por fuera del cuello del tanque, descongelación en la axila, en agua refrigerada y en agua no potable. La integridad acrosómica (IAs) y la integridad estructural de la membrana espermática $(I E M)$ fueron afectadas $(P<0.05)$ por la interacción genotipo x técnica instrumental. La resistencia de las membranas espermáticas fue afectada $(\mathrm{P}<0.01)$ por el genotipo y la técnica instrumental. Los valores más bajos para integridad y resistencia de membranas correspondieron al semen de Pardo Suizo descongelado en la axila y almacenado con bajo nivel de nitrógeno. A pesar de que los porcentajes de gotas citoplásmicas y colas en látigo $(<7 \%$ y $<11.3 \%$, respectivamente) fueron superiores a lo esperado, el porcentaje de espermatozoides normales estuvo siempre por encima del $70 \%$, indicativo de que ninguno de los factores evaluados afectó de manera determinante la morfología espermática.
\end{abstract}

Palabras clave: análisis de semen, espermatozoide bovino, inseminación artificial, preservación de semen. 


\begin{abstract}
This work evaluated cryopreservation at $-196^{\circ} \mathrm{C}$, thawing and semen insemination in 32 bovine herds from the center of Colombia; it also studied, in vitro, the effect of the errors found regarding membrane integrity and sperms morphology. A specific form was used for collecting information from farms. The in vitro study was carried out at the Universidad de Caldas' Agricultural Biotechnology Institute (Instituto de Biotecnología Agropecuaria, Manizales, Colombia); it consisted of using commercial $0.5 \mathrm{ml}$ straws (30x106 epzs) containing sperm from Holstein, Jersey and Brown Swiss bulls, comparing conventional handling technique to five modifications of it (most frequent errors) using a completely randomised $6 \times 3$ factorial design. Artificial insemination (AI) was not used correctly on any of the farms evaluated here. The most frequently occurring errors were deterioration of external structure and inadequate storage conditions for the tanks, storing straws in the bucket and manipulating straws outside the tank neck, thawing in the armpit, in refrigerated water and in non-potable water. Acrosome integrity artificial insemination, semen preservation.

$(A s I)$ and sperm membrane integrity $(\mathrm{SMI})$ were affected $(\mathrm{p}<0.05)$ by genotype $\mathrm{x}$ handling technique interaction. Sperm membrane resistance was affected $(p<0.01)$ by genotype and handling technique. Brown Swiss semen thawed in the armpit and stored with low nitrogen level had the lowest values for membrane integrity and resistance. In spite of cytoplasmic droplet and coiled tail percentages being greater than expected $(<7 \%$ and $<11.3 \%$, respectively), normal sperm percentage was always above $70 \%$, indicating that none of the factors evaluated here decisively affected sperm morphology.
\end{abstract}

Key words: semen analysis, bovine spermatozoa

\begin{abstract}
Resumo
O trabalho avalio a criopreservação a $-196^{\circ} \mathrm{C}$, o descongelamento e a aplicação do sêmen, em 32 rebanhos bovinos do interior da Colômbia, e foi estudada, in vitro, o efeito dos erros encontrados na integridade da membrana e a morfologia do esperma. A recopilação da informação nas fazendas foi realizada por formulário especifico. O estúdio in vitro se executo no Instituto de Biotecnologia Agropecuária da Universidad de Caldas (Manizales, Colômbia), e consistiu em expor palhas comercia-lhes de $0.5 \mathrm{ml}\left(30 \times 10^{6}\right.$ epzs), de toros Holstein, Jersey e Pardo Suíço, à técnica instrumental convencional e a cinco modificações da mesma (erros mais frequentes), com um experimento fatorial $6 \times 3$ com desenho completamente ao acaso. Em nenhuma das fazendas avaliadas se pratico acertadamente a pratica da inseminação artificial (IA). Os erros mais frequentes fórum: deterioro da estrutura externa e condições inadequadas de armazenamento dos tanques, armazenamento de palhas no balde e manuseio de palhas fora do pescoço do tanque, descongelando na axila, água gelada e sem água potável. A integridade acrossômica (IAs) e da integridade estrutural da membrana espermática (IEM) foram influenciados $(\mathrm{P}<0,05)$ pela interação genótipo $\mathrm{x}$ técnica instrumental. A resistência da membrana espermática foi afetada $(\mathrm{P}<0,01)$ pelo genótipo e a técnica instrumental. Os valores mais baixos para a integridade e resistência das membranas correspondeu ao sémen de Pardo Suíço descongelado na axila e armazenado com baixo teor de nitrogênio. Embora as percentagens de gotas citoplasmáticas e caudas em látego $(<7 \%$ e $<11,3 \%$, respectivamente) foram mais elevadas do que se esperava, a percentagem de células normais de esperma foi sempre superior a $70 \%$, indicando que nenhum dos fatores avaliadas influenciarem decisivamente a morfologia espermática.
\end{abstract}

Palavras-chave: espermograma, esperma bovino, inseminação artificial, preservação do sêmen.

\section{Introducción}

La IA es la biotecnología reproductiva más empleada en la industria animal. En la ganadería bovina, su implementación se consolidó a partir del establecimiento del método de fijación cervical, de la aplicación de pequeñas dosis de semen diluido en el cuerpo del útero mediante instrumentación específica, y de la utilización de semen congelado-descongelado (Foote, 2002). La aplicación de esta biotecnología, conllevó a un mejoramiento sustantivo de la productividad; sin embargo, las fallas en la elección del material genético y en la práctica instrumental, afectan de manera grave los indicadores productivos, bien por detrimento en la fertilidad, o por la diseminación de genes indeseables (Nur et al., 2006; Pace et al., 1981).

La evaluación de la fertilidad de un macho, y la manera como es afectada por la manipulación y la descongela- ción del semen criopreservado, requiere de una mayor comprensión de la calidad seminal y de los factores ambientales involucrados (Barth y Waldner, 2002; Saacke, 2003). La reducción de la calidad del semen congelado de bovino guarda relación con fallas en la espermatogénesis y en la manipulación (Barth y Waldner, 2002). Al respecto, las alteraciones en la espermatogénesis, de acuerdo con Spitzer (2000), pueden ser causadas por factores genéticos, nutricionales, tóxicos, relacionados con la edad y estresantes; mientras que las fallas en la manipulación del semen se derivan de los protocolos de congelación y descongelación, y no de la permanencia a temperatura de almacenamiento a $-196{ }^{\circ} \mathrm{C}$ (Correa et al., 1996; Pickett et al., 1978) .

El mantenimiento del semen a $-196^{\circ} \mathrm{C}$ detiene todos los procesos metabólicos, lo que permite su conservación de manera indefinida (Nebel, 1997). Sin embargo, tan- 
to en la criopreservación como en la descongelación se pueden presentar errores que inducen alteraciones en las membranas celulares, los organelos y la interacción célula-célula, que a la postre afectan la capacidad fecundante del espermatozoide (Woods et al., 2004). El retorno a las condiciones fisiológicas que ocurre durante la descongelación, es crítico para la supervivencia celular (Mazur, 1984), y los pasos involucrados en la descongelación exigen máximo cuidado con el tiempo, la temperatura y la manipulación de la pajilla; así por ejemplo, la descongelación de pajillas por más de 30 segundos en temperaturas mayores a $\operatorname{los} 37^{\circ} \mathrm{C}$ redundará en mayor número de alteraciones espermáticas (Correa et al., 1996; Rodriguez et al., 1975), y en porcentajes más altos de retorno al celo (Almquist et al., 1982; Pace et al., 1981; Pickett et al., 1978). Alteraciones similares suceden, cuando se manipulan las pajillas por fuera del cuello del tanque, o cuando los niveles de nitrógeno líquido son insuficientes para mantener la temperatura de $-196^{\circ} \mathrm{C}$ (Berndtson y Pickett, 1978). Además, las bajas temperaturas están asociadas con el aumento de la rigidez de la membrana espermática, y con la reducción de la velocidad del movimiento de moléculas a través de la membrana, mediante los procesos de transporte activo, que terminan sometiendo la célula a estrés osmótico (Mendoza et al., 2000; Watson, 2000) o a shock por frío, con los consecuentes cambios en la estructura del acrosoma (Brown et al., 1982; Nur et al., 2006).

El presente trabajo se realizó con el propósito de detectar las principales errores en la ejecución de la técnica de inseminación artificial con semen congelado en 32 ganaderías bovinas del centro de Colombia, para replicarlas en condiciones experimentales de laboratorio y medir su efecto sobre la integridad de las membranas y la morfología del espermatozoide.

\section{Materiales y métodos}

En el presente trabajo se analizaron las condiciones de criopreservación a $-196{ }^{\circ} \mathrm{C}$, descongelación y aplicación del semen, en 32 ganaderías bovinas del centro de Colombia (Departamentos de Caldas, Santander, Boyacá y Antioquia). El análisis de las condiciones de criopreservación a $-196{ }^{\circ} \mathrm{C}$, se basó en la evaluación de las condiciones de almacenamiento del tanque de criopreservación, del nivel de nitrógeno líquido, y del estado de las principales partes del tanque: tapa, canastillas, escalerillas, cubierta externa y regla para verificación del nivel de nitrógeno. Las condiciones de manipulación, descongelación y aplicación del semen, se registraron directamente en el momento de la actuación de cada uno de 32 inseminadores. La re- colección de la información en las fincas se realizó mediante el diligenciamiento de un formulario elaborado y probado con fines específicos.

Una vez analizados los 32 formularios se seleccionaron los errores más relevantes, con el propósito de establecer su efecto sobre la integridad de las membranas espermáticas y la morfología del espermatozoide, mediante modelo experimental in vitro, desarrollado en el Instituto de Biotecnología Agropecuaria de la Universidad de Caldas (Manizales, Colombia). El material experimental usado fueron pajillas comerciales de $0.5 \mathrm{ml}\left(30 \times 10^{6}\right.$ epzs), de toros Holstein, Jersey y Pardo Suizo. Se probaron seis técnicas instrumentales (TI), diseñadas para simular los errores detectados en finca, a saber:

T I 1. Protocolo convencional: extracción de las pajillas del tanque en menos de 10 segundos y descongelación en agua a $37^{\circ} \mathrm{C}$ durante 30 segundos. Antes de vaciar la pajilla se secó con servilleta estéril y su contenido (500ul) se utilizó para el análisis in vitro.

T I 2. Descongelación en la axila: la descongelación de las pajillas se realizó en la axila durante 180 segundos, antes de utilizar su contenido para el análisis in vitro.

T I 3. Descongelación de la pajilla en agua a $42{ }^{\circ} \mathrm{C}$ : la temperatura del agua para descongelar la pajilla fue de $42{ }^{\circ} \mathrm{C}$ durante 30 segundos, antes de utilizar su contenido para el análisis in vitro.

T I 4. Intervalo descongelación/aplicación de 180 segundos: la pajilla puesta en el inyector (pistola) se dejó expuesta al medio ambiente durante 180 segundos, antes de utilizar su contenido para el análisis in vitro.

TI 5. Extracción incorrecta de la pajilla: la canastilla y la escalerilla con las pajillas, fueron expuestas por fuera del cuello del tanque de criopreservación, durante 30 segundos, tres veces diarias, cuatro días consecutivos, antes de utilizar su contenido para el análisis in vitro.

T I 6. Criopreservación en bajo nivel de nitrógeno líquido: las pajillas se dejaron durante 10 días en un tanque de conservación de 20 litros, con un nivel de nitrógeno líquido de $6 \mathrm{~cm}(6 \mathrm{~L})$, o sea, $4 \mathrm{~cm}(4 \mathrm{~L})$ menos de lo recomendado.

La descongelación de las pajillas en las técnicas 4, 5 y 6 se realizó según el protocolo convencional, es decir, a $37^{\circ} \mathrm{C}$ por 30 segundos (Nur et al., 2006; Pickett et al., 1978). En los seis protocolos, una vez realizada la descongelación de la pajilla, se diluyó el semen en $3.5 \mathrm{ml}$ de Phosphate buffered saline (PBS) a $37^{\circ} \mathrm{C}$, se 
centrifugó a 500 g durante 5 minutos, y se eliminó el sobrenadante. Este proceso se realizó dos veces; el sedimento se utilizó para realizar el análisis in vitro (Brackett y Oliphant, 1975; Risopatron et al., 1994).

La evaluación de la integridad estructural de la membrana plasmática (IEM) se realizó mediante tinción con Eosina-Nigrosina (E/N) (Campbell et al., 1953; Diaz et al., 2009), y evaluación en microscopio de campo claro con objetivo de 40X. Se reportó el porcentaje de espermatozoides vivos.

La IAs fue evaluada mediante fijación con glutaraldehido al $2 \%$ en Beltsville Thawing Solution (BTS) (Osorio-Serna et al., 2007; Pursel y Johnson, 1974), y evaluación en microscopio de contraste de fases con objetivo de 40X. Se reportó el porcentaje de espermatozoides con la cresta apical normal.

La resistencia de las membranas acrosómica y espermática (RMs), se evaluó mediante la técnica desarrollada por Perez-Llano et al., (2003) que consiste en la combinación de un short hypoosmotic swelling test (sHOST) y una fijación posterior en glutaraldehído al $2 \%$. La preparación se evaluó en microscopio de contraste de fases con objetivo de 40X. Se reportaron los porcentajes de tres categorías celulares así: espermatozoides con acrosoma intacto (membrana acrosómica resistente) y sHOST tanto positivo como negativo, espermatozoides con sHOST positivo independiente del estado del acrosoma (membrana espermática resistente), y espermatozoides sHOST positivo con el acrosoma intacto (membranas espermática y acrosómica resistentes).

La morfología espermática fue evaluada en semen fijado con glutaraldehido al $2 \%$ en BTS, mediante microscopio de contraste de fases con objetivo de 40X. Se reportó el porcentaje de espermatozoides normales y de espermatozoides con malformaciones de cabeza (macrocabeza, microcabeza, piriforme y suelta), tracto intermedio (doble, engrosado, lazo y excéntrico) y cola (látigo, lazo, ovillo, escalera y suelta) y con gota citoplásmica (Kasimanickam et al., 2006).
Se realizó un arreglo factorial $6 \times 3$ (6 técnicas instrumentales y 3 genotipos bovinos) en un diseño completamente al azar, con una pajilla como unidad experimental y 2 réplicas por tratamiento. Todos los resultados se analizaron con el programa SAS Inst. Inc.; cory.NC. Se utilizó el programa proc GLM para los efectos probados y se realizó una prueba de comparaciones múltiples mediante la prueba de Tukey.

\section{Resultados}

\section{Condiciones físicas de la criopreservación}

De los 32 tanques observados, se encontraron 12 con alteraciones de sus componentes, así: tres $(9.37 \%)$ con daños en la tapa, dos $(6.25 \%)$ en la canastilla, cuatro $(12.5 \%)$ en la parte externa y cuatro $(12.5 \%)$ en la regla de medición. En cuatro de las fincas evaluadas, se encontraron varias irregularidades de los tanques que, de manera simultánea, afectaron la tapa, la parte externa, el estado de las canastillas y la regla de medición (Tabla 1).

En cuanto al sitio de almacenamiento, ocho (25\%) de los 32 tanques analizados se encontraron almacenados incorrectamente: seis $(18.75 \%)$ en sitios con mala ventilación, dos (6.25\%) expuestos a la acción directa del sol, y uno presentó ambas irregularidades. Es de resaltar, que los cuatro tanques que registraron problemas en su estructura externa, también presentaron condiciones inadecuadas de almacenamiento.

Al revisar los 32 tanques, se encontró que 31 contenían en promedio $21.93 \mathrm{~cm}$ de nitrógeno líquido, con una frecuencia de revisión del nivel de nitrógeno de 50.34 días, en promedio, en solo uno (3.13\%), se encontró el nivel de nitrógeno por debajo del mínimo recomendado $(10 \mathrm{~cm}$ de altura o $10 \mathrm{~L}$ de nitrógeno) $y$ en inadecuadas condiciones de almacenamiento, $y$ en solo uno $(3.13 \%)$ se conservaron las pajillas en la canastilla.

Con relación a los 32 inseminadores analizados, 17 $(53.13 \%)$ se tardaron 24.34 segundos en promedio

Tabla 1. Condiciones físicas de 32 tanques de criopreservación de semen bovino en la región central de Colombia

\begin{tabular}{|l|c|c|c|c|c|}
\hline \multicolumn{7}{|c|}{ Componentes del tanque / Porcentaje (valor absoluto) } \\
\hline \multicolumn{1}{|c|}{ Condición } & Tapa & Canastilla & Escalerilla & Parte externa & Regla \\
\hline Buena & $90.63(29)$ & $93.75(30)$ & $100(32)$ & $87.50(28)$ & $87.50(28)$ \\
\hline Regular & $9.37(3)$ & $6.25(2)$ & $0(0)$ & $12.50(4)$ & $9.37(3)$ \\
\hline Mala & $0(0)$ & $0(0)$ & $0(0)$ & $0(0)$ & $3.13(1)$ \\
\hline
\end{tabular}


para extraer la pajilla y $15(46.87 \%)$ la extrajeron correctamente, es decir, en un tiempo máximo de 15 segundos; adicionalmente, 20 (62.5\%) manipularon la pajilla dentro del cuello del tanque y doce $(37.5 \%)$ por fuera del mismo.

\section{Descongelación y posterior manipulación del semen}

Con relación al procedimiento de descongelación, de los 32 inseminadores, 25 realizaron la descongelación de la pajilla en agua caliente: nueve $(36 \%)$ descongelaron a $37^{\circ} \mathrm{C}, 12(48 \%)$ lo hicieron entre $35^{\circ} \mathrm{C}$ y $37^{\circ} \mathrm{C}$ en tiempos variables, y solo cuatro $(16 \%)$ realizaron la descongelación a $37{ }^{\circ} \mathrm{C}$ durante 30 segundos. Por otra parte, de los 29 inseminadores que descongelaron en agua, $13(44.83 \%)$ lo hicieron en recipientes sin tapa, 25 inseminadores $(86.2 \%)$ utilizaron agua no potable para la descongelación, nueve (28.12\%) no secaron la pajilla antes de montarla en la pistola de inseminación, cuatro $(12.5 \%)$ descongelaron en agua refrigerada a temperaturas entre $4{ }^{\circ} \mathrm{C}$ y $5{ }^{\circ} \mathrm{C}$ por 60 a 160 segundos y tres $(9.38 \%)$ descongelaron la pajilla en la axila del inseminador.

En cuanto al intervalo descongelación/aplicación, ocho $(25 \%)$ de los inseminadores evaluados, se tomaron alrededor de cinco minutos.

\section{Integridad de las membranas espermáticas}

La IAs y la IEM fueron afectadas de manera significativa $(\mathrm{P}<0.05)$ por la interacción genotipo $\mathrm{x}$ técnica instrumental (Tabla 2). El semen de toros Pardo Suizo descongelado en la axila o sometido a bajo nivel de nitrógeno, presentó los valores mas bajos para IAs, $33.5 \%$ y $40.2 \%$ respectivamente. Así mismo, se evidenció que el semen de toros Holstein registró los valores más altos de IAs, comprendidos entre $54.7 \%$ y $80.2 \%$ (Fig. 1). En semen de toros Pardo suizo sometido a bajo nivel de nitrógeno líquido, se encontró un valor significativamente inferior $(\mathrm{P}<0.05)$ para IEM (47\%) (Fig. 1). Con relación a las 17 combinaciones restantes (genotipo $\mathrm{x}$ técnica instrumental) no registraron diferencias significativas para estas variables $(\mathrm{P}>0.05)$.

\section{Resistencia de las membranas espermáticas}

La resistencia de las membranas espermáticas fue afectada de manera altamente significativa $(\mathrm{P}<0.01)$ por el genotipo y la técnica instrumental (Tabla 2), encontrándose que los valores promedio más bajos de resistencia de membranas (RMs), resistencia acrosómica (RA) y resistencia de la membrana espermática (RME), fueron $14.6 \%, 19.7 \%$, y $15.4 \%$, respectivamente, y se obtuvieron cuando se descongeló el semen en la axila (Fig. 2).

Con respecto al genotipo, el semen de toros Pardo suizo mostró mayor sensibilidad a los efectos de las

Tabla 2. Efecto de la técnica instrumental $(\mathrm{TI})$, del genotipo $(\mathrm{G})$ y de la interacción entre estos dos factores $(\mathrm{G} \times \mathrm{TI})$ sobre la calidad seminal en semen bovino congelado-descongelado

\begin{tabular}{|c|c|c|c|}
\hline \multirow{2}{*}{ Variable } & \multirow{2}{*}{ T I } & Fc & \multirow{2}{*}{ TI $\times$ G } \\
\hline & & G & \\
\hline Integridad acrosómica (IAs) & $14.96^{* *}$ & $53.19^{* *}$ & $8.68^{* *}$ \\
\hline Integridad estructural de la membrana espermática (IEM, \#109) & $1.60^{N S}$ & $12.02^{* *}$ & $2.99^{*}$ \\
\hline Resistencia de membranas acrosómica y espermática (RMs) & $9.08^{* *}$ & $33.66^{* *}$ & $0.65^{N S}$ \\
\hline Resistencia de la membrana acrosómica (RA) & $7.21^{* *}$ & $33.25^{* *}$ & $0.64^{N S}$ \\
\hline Resistencia de la membrana espermática (RME) & $9.74^{* *}$ & $34.52^{* *}$ & $0.44^{N S}$ \\
\hline Porcentaje de espermatozoides normales (PEN) & $2.61^{N S}$ & $1.39^{N S}$ & $0.87^{N S}$ \\
\hline Colas en látigo $(\mathrm{CL})$ & $3.38^{*}$ & $14.24^{* *}$ & $0.63^{N S}$ \\
\hline Gotas citoplásmicas (GCs) & $0.78^{N S}$ & $1.28^{N S}$ & $1.36^{N S}$ \\
\hline
\end{tabular}

NS: $\quad$ Efecto no significativo $(P>0.1)$;

*: $\quad$ Efecto significativo $(P \leq 0.05)$;

**: $\quad$ Efecto altamente significativo $(P \leq 0.01)$ 


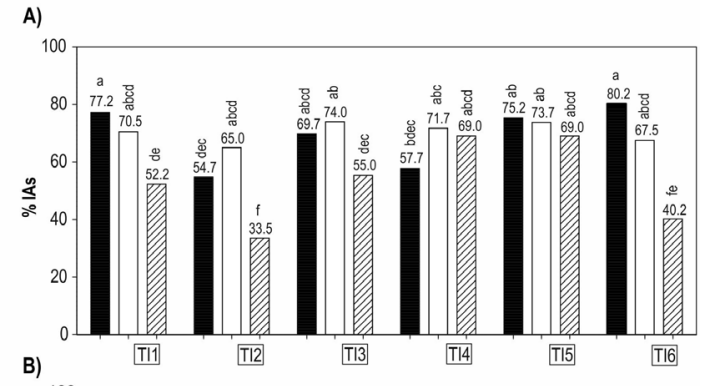

B)

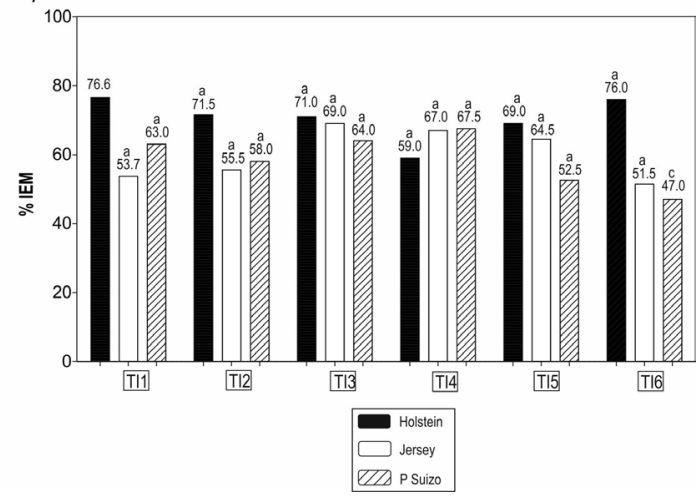

Figura 1. Comparación de medias para integridad acrosómica (A) e integridad estructural de membrana (B), bajo la interacción genotipo $x$ técnica instrumental, mediante la prueba de Tukey. TI 1: protocolo convencional, TI 2: descongelación en la axila, TI 3: descongelación en agua a $42^{\circ} \mathrm{C}$, TI 4: intervalo descongelación/aplicación de 180 segundos, TI 5: extracción incorrecta de la pajilla (fuera del tanque de criopreservación durante 30 segundos por 3 veces), TI 6: bajo nivel de nitrógeno líquido. Medias identificadas con letras iguales, fueron iguales para la prueba de Tukey $(\mathrm{P}>0.05)$.
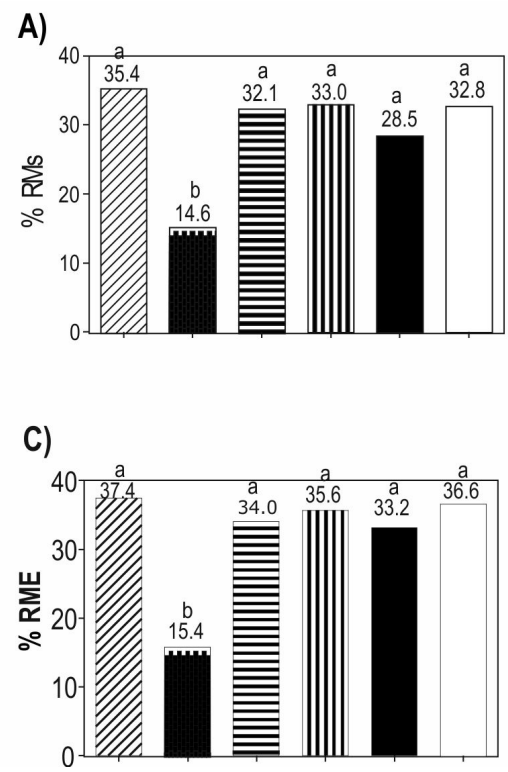

técnicas instrumentales sobre estas variables, con valores por debajo del 25\% (Fig. 3).

\section{Morfología espermática}

Con relación a la morfología espermática, las malformaciones de cabeza, tracto intermedio y cola (excepto colas en látigo) no fueron reportadas debido a su nula o baja presentación. En cuanto a las colas en látigo $(\mathrm{CL})$, se encontró que fueron afectadas de manera significativa $(\mathrm{P}<0.05)$ por la técnica instrumental y de manera altamente significativa $(\mathrm{P}<0.01)$ por el genotipo (Tabla 2); sin embargo, en la prueba de Tukey, los valores promedio obtenidos en esta variable, por efecto de la técnica instrumental, fueron estadísticamente iguales ( $\mathrm{P}>0.05)$ (Fig. 4). El semen de Holstein y Jersey registró valores para $\mathrm{CL}$ estadísticamente iguales ( $P>0.05$ ) (12.50\% y 9.1\%), mientras el de Pardo Suizo presentó valores significativamente inferiores $(\mathrm{P}<0.05)$ para esta alteración (4\%) (Fig. 4).

\section{Discusión}

\section{Condiciones físicas de la criopreservación}

Los hallazgos encontrados en cuatro (12.5\%) de los tanques evaluados, podrían provocar una perdida exagerada de nitrógeno líquido y generar un riesgo

B)

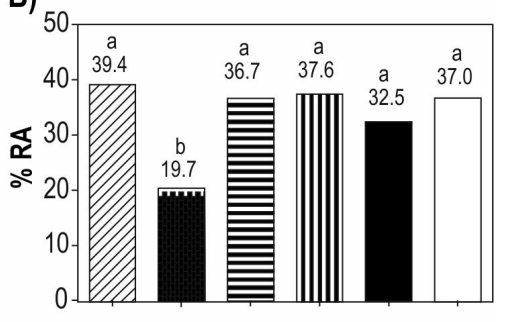

Figura 2. Comparación de medias para la resistencia de membranas (A), resistencia acrosómica (B), y resistencia de la membrana espermática (C), bajo seis técnicas instrumentales, mediante la prueba de Tukey. TI 1: protocolo convencional, TI 2: descongelación en la axila, TI 3: descongelación en agua a $42^{\circ} \mathrm{C}$, TI 4: intervalo descongelación/aplicación de 180 segundos, TI 5: extracción incorrecta de la pajilla (fuera del tanque de criopreservación durante 30 segundos por 3 veces), TI 6: bajo nivel de nitrógeno líquido. Medias identificadas con letras iguales, fueron iguales para la prueba de Tukey $(\mathrm{P}>0.05)$. 
A)

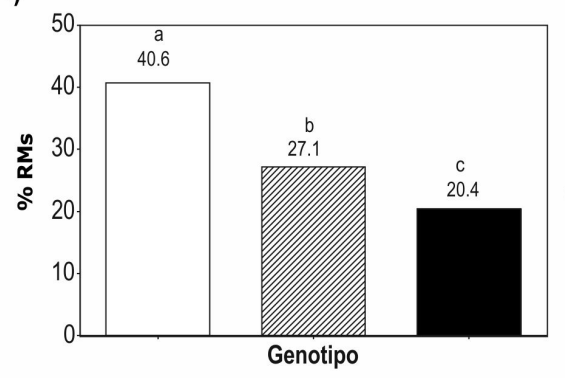

C)

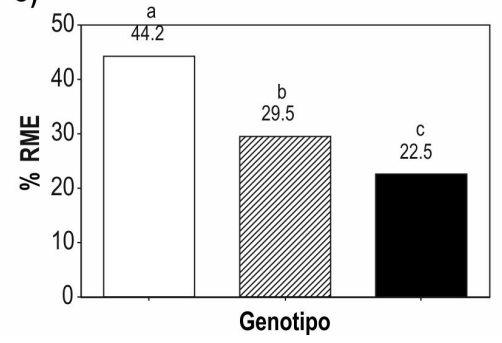

B)
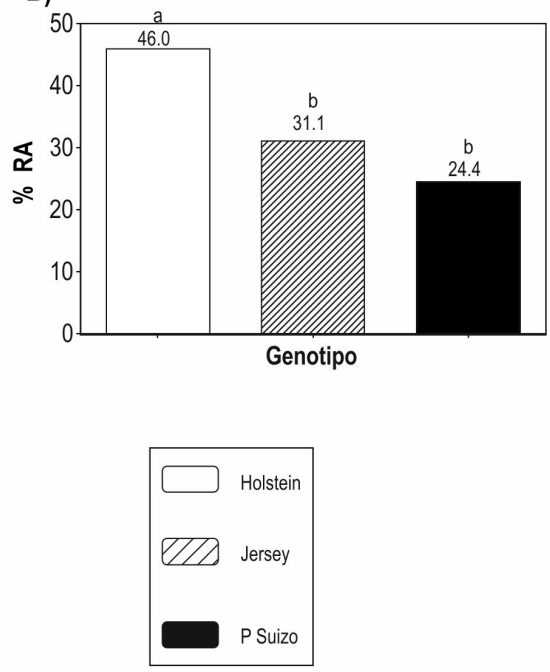

Figura 3. Comparación de medias para la resistencia de membranas (A), resistencia acrosómica (B) y resistencia de la membrana espermática (C), bajo tres genotipos del género bos taurus, mediante la prueba de Tukey. Medias identificadas con letras iguales, fueron iguales para la prueba de Tukey $(\mathrm{P}>0.05)$.
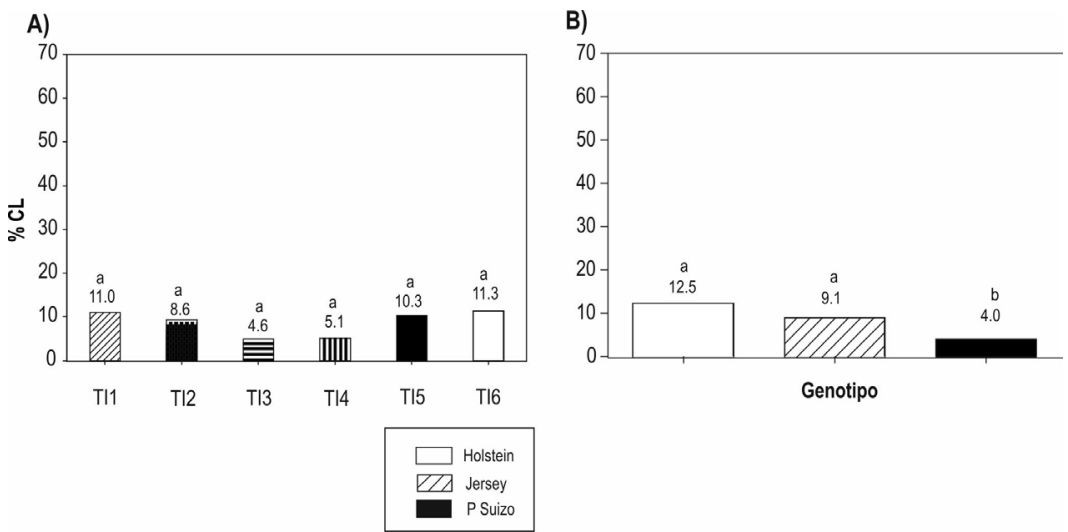

Figura 4. Comparación de medias para cola en látigo bajo seis técnicas instrumentales $(\mathrm{A})$ y tres genotipos del género bos taurus (B), mediante la prueba de Tukey. TI 1: protocolo convencional, TI 2: descongelación en la axila, TI 3: descongelación en agua a $42^{\circ} \mathrm{C}$, TI 4: intervalo descongelación/aplicación de 180 segundos, TI 5: extracción incorrecta de la pajilla (fuera del tanque de criopreservación durante 30 segundos por 3 veces), TI 6: bajo nivel de nitrógeno líquido. Medias identificadas con letras iguales, fueron iguales para la prueba de Tukey $(\mathrm{P}>0.05)$.

inminente de incorrecta criopreservación del semen (Ávila-Portillo et al., 2006).

En cuanto al sitio de almacenamiento del tanque se consideraron las recomendaciones hechas por Castillo et al., (2002): buena ventilación, temperatura ambiental inferior a $50{ }^{\circ} \mathrm{C}$ y protección a la exposición directa a los rayos solares; al respecto, ocho $(25 \%)$ de los 32 tanques analizados se encontraron almacenados incorrectamente: seis $(18.75 \%)$ en sitios con mala ventilación, dos (6.25\%) expuestos a la acción directa del sol, y uno presentó ambas irregularidades. Es de resaltar, que los cuatro tanques que registraron pro- blemas en su estructura externa, también presentaron condiciones inadecuadas de almacenamiento; lo cual, según Ávila-Portillo et al., (2006) ocasiona disminución drástica del nivel de nitrógeno y altera la adecuada criopreservación del semen.

Al revisar los 32 tanques solo uno (3.13\%), se encontró el nivel de nitrógeno por debajo del mínimo recomendado y en inadecuadas condiciones de almacenamiento. Todas las fallas detectadas en los tanques de conservación están asociadas con dificultades para mantener un nivel de nitrógeno constante y adecuado, que afectan la integridad acrosómica, la integridad fun- 
cional de la membrana, la movilidad y la supervivencia espermática (Nur et al., 2006), debido a que en el interior del tanque no se logra mantener la temperatura de $-196^{\circ} \mathrm{C}$, sometiendo, por tanto, a los espermatozoides a condiciones que generan lesiones en la membrana espermática por fenómenos de recristalización (Nebel, 1997; Nur et al., 2006; Pickett et al., 1978).

La forma como se almacenan las pajillas dentro del tanque de conservación, el tiempo que este permanece destapado al momento de la extracción, la manipulación de la canastilla y la escalerilla respecto al nivel del cuello, así como la temperatura y el tiempo de descongelación de las pajillas, son características que de manera individual o colectiva, influyen sobre la calidad espermática (Pace et al., 1981). Al respecto, de los 32 tanques analizados, en solo uno (3.13\%) se conservaron las pajillas en la canastilla, hecho que dificulta la manipulación y el tiempo de extracción de las mismas, lo que genera cambios bruscos en la temperatura y por ende disminución de la movilidad espermática (Nebel, 1997). Con relación a los 32 inseminadores analizados, 17 (53.13\%) se tardaron 24.34 segundos en promedio para extraer la pajilla y $15(46.87 \%)$ la extrajeron correctamente, es decir, en un tiempo máximo de 15 segundos (Nebel, 1997); adicionalmente, $20(62.5 \%)$ manipularon la pajilla dentro del cuello del tanque y doce $(37.5 \%)$ por fuera del mismo; esta práctica genera alteraciones en la movilidad y la supervivencia espermática, debido a que al someter el semen a temperaturas comprendidas entre $-79^{\circ} \mathrm{C}$ y $-100^{\circ} \mathrm{C}$ se genera un fenómeno de recristalización de la membrana, que la lesiona irreversiblemente (Nur et al., 2006).

\section{Descongelación y aplicación del semen}

Con relación al procedimiento de descongelación, de los 32 inseminadores, solo cuatro $(16 \%)$ realizaron la descongelación a $37^{\circ} \mathrm{C}$ durante 30 segundos, combinación que según Correa et al., (1996) y Pace et al., (1981) se asocia con buen funcionamiento e integridad de la membrana espermática, y correcta movilidad. Por otra parte, de los 29 inseminadores que descongelaron en agua, 13 (44.83\%) lo hicieron en recipientes sin tapa, lo cual puede afectar el mantenimiento de la temperatura de descongelación y consecuentemente la calidad seminal (Nebel, 1997).

Montar la pajilla mojada en la pistola de inseminación puede generar contacto de las células espermáticas con agua, hecho que causa lesiones irreversibles sobre la supervivencia espermática por cambios en la osmolaridad (Nebel, 1997); en las observaciones de campo de este trabajo, 25 inseminadores (86.2\%) utilizaron agua no potable para la descongelación y nueve
$(28.12 \%)$ no secaron la pajilla antes de montarla en la pistola de inseminación. De otro lado, cuatro de los inseminadores $(12.5 \%)$ descongelaron en agua refrigerada a temperaturas entre $4^{\circ} \mathrm{C}$ y $5^{\circ} \mathrm{C}$ por 60 a 160 segundos; esta práctica genera ruptura de la membrana espermática (Nur et al., 2006) y disminuye la proporción de espermatozoides con acrosoma intacto (Almquist et al., 1982), con movilidad progresiva y con actividad de la acrosina (Pace et al., 1981). Adicionalmente, tres inseminadores $(9.38 \%)$ descongelaron la pajilla en la axila, práctica que produce variaciones de temperatura y conduce a daño de la membrana espermática y a una excesiva producción de radicales libres (O'Flaherty et al., 2003).

Ocho $(25 \%)$ de los inseminadores evaluados se tomaron alrededor de cinco minutos de intervalo descongelación/aplicación, o sea estuvieron dentro del rango recomendado por Nebel (1997) y Troxel (2007), consistente en que cada pajilla debe ser utilizada en los primeros quince minutos después de descongelada, para evitar variaciones de temperatura y el consumo de las reservas energéticas del semen.

Los resultados de la evaluación de las condiciones de criopreservación a $-196^{\circ} \mathrm{C}$, congelación y aplicación del semen en 32 ganaderías bovinas del centro de Colombia, permitieron establecer que en ninguna de las fincas evaluadas se ejecutó correctamente la práctica de la inseminación artificial, debido a que siempre se detectaron errores en por lo menos una de las condiciones. Los hallazgos anteriores, señalaron la necesidad de establecer el efecto de las condiciones de criopreservación a $-196^{\circ} \mathrm{C}$, descongelación y aplicación del semen sobre la morfofisiología seminal in vitro.

\section{Integridad de las membranas espermáticas}

El semen de toros Pardo Suizo descongelado en la axila o sometido a bajo nivel de nitrógeno, presentó los valores mas bajos para IAs, $33.5 \%$ y $40.2 \%$ respectivamente; estos resultados concuerdan con lo reportado por Nur et al. (2006), quienes encontraron una reducción significativa en la IAs por exposición de semen de toros Holstein a bajos niveles de nitrógeno. En semen de toros Pardo suizo sometido a bajo nivel de nitrógeno líquido, se encontró un valor significativamente inferior $(\mathrm{P}<0.05)$ para IEM $(47 \%)$ (Fig. 1$)$, el cual estuvo por debajo de lo reportado por Rubio-Guillen et al., (2007) en semen congelado-descongelado de toros adultos; al respecto, Nur et al., (2006), afirman que el bajo nivel de nitrogeno líquido afecta la integridad acrosómica, la integridad funcional y estructural de la membrana y la movilidad. 


\section{Resistencia de las membranas espermáticas}

En el experimento in vitro los valores promedio más bajos de resistencia de membranas (RMs), resistencia acrosómica (RA) y resistencia de la membrana espermática (RME), se obtuvieron cuando se descongeló el semen en la axila (Fig. 2). Estos cambios pueden ser explicados porque al modificar la temperatura y el tiempo de descongelación $\left(37^{\circ} \mathrm{C}\right.$ y 30 segundos), se generan especies de oxígeno reactivas y consecuentemente peroxidación lipídica, lo cual afecta la integridad estructural y funcional de la membrana y en especial la movilidad total y progresiva de los espermatozoides (Anzar et al., 2011).

Con respecto al genotipo, el semen de toros Pardo suizo mostró mayor sensibilidad a los efectos de las técnicas instrumentales sobre la resistencia de las membranas espermáticas, con valores por debajo del 25\% (Fig. 3). De igual manera, todos los valores estubieron por debajo de lo encontrado por Rubio-Guillen et al. (2007), quienes sometieron el semen a la prueba de resistencia osmótica con el fin de predecir su fertilidad y congelabilidad, encontrando valores de $52,2 \%$.

\section{Morfología espermática}

Las colas en látigo son alteraciones que se generan durante el transito por el epidídimo, y las GCs se observan con mayor frecuencia en toros jóvenes (AlMakhzoomi et al., 2008; Carreira et al., 2012); a pesar de que en este trabajo los porcentajes de GCs y CL estuvieron por encima de lo encontrado en investigaciones afines (Hallap et al., 2004; Spitzer, 2000; Veznik et al., 2007), el porcentaje de espermatozoides normales estuvo siempre por encima del $70 \%$.

En todas las variables analizadas, el semen de toros Hosltein tuvo valores superiores al semen de toros Jersey y Pardo suizo, lo cual concuerda con lo reportado por Kasimanickam et al., (2006), quienes al realizar una comparación entre semen de toros Jersey y Holstein, encontraron en este ultimo genotipo, una menor tendencia de fragmentación del ADN, así como una menor afección de la integridad de la membrana plasmática.

\section{Conclusiones}

Los resultados de la evaluación de las condiciones de criopreservación a $-196^{\circ} \mathrm{C}$, congelación y aplicación del semen en 32 ganaderías bovinas del centro de Colombia, permitieron establecer que en ninguna de las fincas evaluadas se ejecutó correctamente la práctica de la inseminación artificial, debido a que siempre se detectaron errores en por lo menos una de las condiciones.

La integridad acrosómica (IAs) y la integridad estructural de la membrana espermática (IEM) fueron afectadas de manera significativa $(\mathrm{P}<0.05)$ por la interacción genotipo $x$ técnica instrumental. El semen de toros Pardo Suizo descongelado en la axila o sometido a bajo nivel de nitrógeno, presentó los valores mas bajos para IAs, $33.5 \%$ y $40.2 \%$ respectivamente; del mismo modo, presentó valores significativamente inferiores $(\mathrm{P}<0.05)$ para IEM $(47 \%)$, cuando se sometió a bajo nivel de nitrógeno.

La resistencia de las membranas espermáticas fue afectada de manera altamente significativa $(\mathrm{P}<0.01)$ por el genotipo y la técnica instrumental, los valores promedio más bajos de resistencia de membranas (RMs), resistencia acrosómica (RA) y resistencia de la membrana espermática (RME), fueron 14.6\%, 19.7\%, y $15.4 \%$, respectivamente, y se obtuvieron cuando se descongeló el semen en la axila.

A pesar de que los porcentajes de GCs y CL $(<7 \%$ y $<11.3 \%$, respectivamente) estuvieron por encima de lo encontrado en investigaciones afines, el porcentaje de espermatozoides normales estuvo siempre por encima del $70 \%$, lo cual fue indicio de que ninguno de los factores evaluados (genotipo, técnica instrumental, y su interacción) afectó de manera determinante la morfología espermática.

Este trabajo ha permitido confirmar la mala ejecución de la técnica de inseminación artificial en condiciones de campo y establecer que las fallas que más afectaron la calidad seminal fueron la descongelación en la axila del inseminador y todo aquello que impide mantener adecuados niveles de nitrógeno líquido en los tanques de criopreservación.

\section{Referencias}

Al-Makhzoomi A, Lundeheim N, Haard M, Rodriguez-Martinez $\mathrm{H}$. Sperm morphology and fertility of progeny-tested Al dairy bulls in Sweden. Theriogenology. 2008;70(4):682-691.

Almquist JO, Grube KE, Rosenberger JL. Effect of thawing time on fertility of bovine spermatozoa in French straws. J Dairy Sci. 1982;65(5):824-827.

Anzar M, Kroetsch T, Boswall L. Cryopreservation of bull semen shipped overnight and its effect on post-thaw sperm motility, plasma membrane integrity, mitochondrial membrane potential and normal acrosomes. Anim Reprod Sci. 2011;126(1-2):23-31.

Ávila-Portillo LM, Madero JI, López C, León MF, Acosta L, et al. Fundamentos de criopreservación. Rev Colomb Obstet Ginecol. 2006;57 (4):291-300. 
Barth AD, Waldner CL. Factors affecting breeding soundness classification of beef bulls examined at the Western College of Veterinary Medicine. The Canadian veterinary journal La revue veterinaire canadienne. 2002;43(4):274-284.

Berndtson WE, Pickett BW. 1978. Thecniques for the cryopreservation and fiel handling of bovine spermatozoa. NAO Sciences editor.

Brackett BG, Oliphant G. Capacitation of rabbit spermatozoa in vitro. Biol Reprod. 1975;12(2):260-274.

Brown JL, Senger PL, Hillers JK. Influence of thawing time and postthaw temperature on acrosomal maintenance and motility of bovine spermatozoa frozen in .5-ml French straws. J Anim Sci. 1982;54(5):938-944.

Campbell RC, Hancock JL, Rothschild L. Counting live and dead bull spermatozoa. J Exp Biol. 1953;30:44-49.

Carreira JT, Mingoti GZ, Rodrigues LH, Silva C, Perri SH, Koivisto MB. Impact of proximal cytoplasmic droplets on quality traits and in-vitro embryo production efficiency of cryopreserved bull spermatozoa. Acta Vet Scand. 2012;54:1.

Castillo R, Morales AM, Carrasco A. Guia de uso de la criocirugia en atencion primaria. Medicina de Familia (And). 2002;3(2);114122.

Correa JR, Rodriguez MC, Patterson DJ, Zavos PM. Thawing and processing of cryopreserved bovine spermatozoa at various temperatures and their effects on sperm viability, osmotic shock and sperm membrane functional integrity. Theriogenology. 1996;46(3):413-420.

Diaz O, Mesa H, Gómez G, Henao FJ. Evaluación invitro de la viabilidad del semen porcino hasta 120 horas de almacenamiento en refrigeración. Veterinaria y Zootecnia. 2009;3:32-37.

Foote $\mathrm{RH}$. The history of artificial insemination: Selected notes and notables. J Anim Sci. 2002;80:1-10.

Hallap T, Haard M, Jaakma U, Larsson B, Rodriguez-Martinez H. Does cleansing of frozen-thawed bull semen before assessment provide samples that relate better to potential fertility? Theriogenolog. 2004;62(3-4):702-713.

Kasimanickam R, Nebel RL, Peeler ID, Silvia WL, Wolf KT, et al. Breed differences in competitive indices of Holstein and Jersey bulls and their association with sperm DNA fragmentation index and plasma membrane integrity. Theriogenology. 2006;66(5):1307-1315.

Mazur P. Freezing of living cells: mechanisms and implications. Am J Physiol. 1984;247(3 Pt 1):C125-142.

Mendoza JA, Dulin P, Warren T. The lower hydrolysis of ATP by the stress protein GroEL is a major factor responsible for the diminished chaperonin activity at low temperature. Cryobiolog. 2000;41(4):319-323.

Nebel RL. 1997. Thechniques for Artificial Insemination of cattle whith frozen-thawed semen. RS Youngquist editor. En: Current therapy in large animal theriogenology p. 251-256.
Nur Z, Kamuran II, Ak K. Effects of different temperature treatments applied to deep stored bull semen on post-thaw cold shocked spermatozoa. Bull Vet Inst Pulawy. 2006;50:79-83.

O'Flaherty C, Beorlegui N, Beconi MT. Participation of superoxide anion in the capacitation of cryopreserved bovine sperm. Int J Androl. 2003;26(2):109-114.

Osorio-Serna RE, Giraldo JF, Mesa H, Gómez G, Henao FJ. Evaluación de la integridad acrosómica en semen de verraco. Veterinaria y Zootecnia. 2007;1:41-47.

Pace MM, Sullivan JJ, Elliott FI, Graham EF, Coulter GH. Effects of thawing temperature, number of spermatozoa and spermatozoal quality on fertility of bovine spermatozoa packaged in 5 ml french straws. J Anim Sci. 1981;53:693-701.

Perez-Llano B, Yenes-Garcia P, Garcia-Casado P. Four subpopulations of boar spermatozoa defined according to their response to the short hypoosmotic swelling test and acrosome status during incubation at 37 degrees C. Theriogenology. 2003;60(8):1401-1407.

Pickett BW, Berndtson WE, Sullivan JJ. Influence of seminal additives and packaging systems on fertility of frozen bovine spermatozoa. J Anim Sci. 1978;47(Suppl 2):12-47.

Pursel VG, Johnson LA. Glutaraldehyde fixation of boar spermatozoa for acrosome evaluation. Theriogenology. 1974; 1(2):63-68.

Risopatron J, Sanchez R, Sepulveda N, Villagran E, Peña P. 1994. Seleccion de espermatozoides de bovino desde semen congelado-descongelado.Comparacion de dos metodos. Archivos de Medicina Veterinaria (Valdivia). 1994;26(1):25-40.

Rodriguez OL, Berndtson WE, Ennen BD, Pickett BW. Effect of rates of freezing, thawing and level of glycerol on the survival of bovine spermatozoa in straws. J Anim Sci. 1975;41(1):129-136.

Rubio-Guillen J, González D, González Y, Madrid-Bury N, QuinteroMoreno A. Puede el ORT complementar las pruebas clasicas de valoracion seminal y predecir la fertilidad en toros. Revista Luz. 2007;1-5.

Saacke R. Fertilidad del toro: una opinion sobre su estado actual y perspectivas. Taurus, BsAs. 2003;5:18-28.

Spitzer J. 2000. Bull Breeding Soundness Evaluation: Current Status. IN Chenoweth P.J. International Veterinary Information Service editor. En: Topics in Bull Fertility. Manhattan, Kansas, USA.

Troxel TR. 2007. Purebred cattle series: artificial insemination. University of Arkansas Division of Agriculture, Cooperative Extension Service, FSA3118.

Veznik Z, Svecova D, Zajicova A, Reckova Z, J. R. The interrelationship between quality parameters of sperm before and after separation by gradient centrifugation. Veterinarni Medicina. 2007;52(10):423-429.

Watson PF. The causes of reduced fertility with cryopreserved semen. Animal reproduction science. 2000;60-61:481-492.

Woods EJ, Benson JD, Agca Y, Critser JK. Fundamental cryobiology of reproductive cells and tissues. Cryobiology. 2004;48(2):146-156. 